\title{
THERMAL PERFORMANCE ANALYSIS OF CONVECTIVE-RADIATIVE FIN WITH TEMPERATURE-DEPENDENT THERMAL CONDUCTIVITY IN THE PRESENCE OF UNIFORM MAGNETIC FIELD USING PARTIAL NOETHER METHOD
}

\author{
M. G. Sobamowo ${ }^{1}$, A. O. Adesina ${ }^{1}$
}

\begin{abstract}
In this paper, thermal performance of convective-radiative straight fin with temperature-dependent thermal conductivity in the presence of uniform magnetic field is analyzed using partial Noether method. The exact analytical solution is used to investigate the effects of magnetic field, convective, radiative, thermo-geometric and thermal conductivity (non-linear) parameters on the thermal performance of the fin. The results reveal that as the magnetic, convective and radiative parameters increase, the temperature of the fin decreases rapidly and by implication, the rate of heat transfer through the fin increases. The study provides a platform for comparison of results of any other method of analysis of the problem with the results of the exact analytical solutions in this paper. Also, such an analytical tool is valuable as a design and optimization approach for large scale (not necessarily in size) finned heat exchangers where each fin/row are analytically analyzed and where the surrounding fluid is influenced by a magnetic field.
\end{abstract}

Keywords: Thermal Performance, Convective-Radiative Fin, Partial Noether Method, TemperatureDependent Thermal Conductivity, Magnetic Field

\section{INTRODUCTION}

The quest for heat transfer enhancement in thermal components and the requirements of high-performance heat transfer equipment and devices that enhance heat dissipation from thermal systems have been subjects of interest over the years. In such quest and requirements, extended surfaces such as fins and spines have been used and the effects of various operating and controlling parameters on the thermal performance of the fins and spines have been investigated. Also, the nonlinearities in the developed thermal model of the passive devices for cooling of thermal systems have attracted a large number of research works. In such thermal analysis, various methods have been applied such regular perturbation expansion [1-2], method of successive approximation [3], Adomian decomposition Method [4-5], homotopy perturbation method [6-10], Homotopy analysis method [11-13], variational iteration method [13-18], Galerkin's method of weighted residual [19]. Differential transform method [20-24] to solve the nonlinear fin problem. The above reviewed approximate analytical methods solve nonlinear differential equations without linearization, without restrictive assumptions or any perturbation, without discretization or approximation of the derivatives. However, most of the approximate methods give accurate predictions only when the nonlinearities are weak and they fail to predict accurate solutions for strong nonlinear models. Also, when they are routinely implemented, they can sometimes lead to erroneous results $[25,26]$. Additionally, some of approximate analytical methods require more mathematical manipulations and they are not applicable to all problems, and thus they suffer a lack of generality. For example, DTM proved to be more effective than most of the other approximate analytical solutions as it does not require many computations as carried out in ADM, HAM, HPM, and VIM. However, the transformation of the nonlinear equations and the development of equivalent recurrence equations for the nonlinear equations using DTM proved somehow difficult in some nonlinear system such as in rational Duffing oscillator, irrational nonlinear Duffing oscillator, finite extensibility nonlinear oscillator. Moreover, the determination of Adomian polynomials as carried out in ADM, the restrictions of HPM to weakly nonlinear problems as established in literatures, the lack of rigorous theories or proper guidance for choosing initial approximation, auxiliary linear operators, auxiliary functions, and auxiliary parameters in HAM and the search Lagrange multiplier as carried in VIM, and the challenges associated with proper construction of the approximating functions for arbitrary domains or geometry of interest as in Galerkin weighted residual method (GWRM), least square method (LSM) and collocation method (CM) are some of the difficulties in applying these approximate analytical methods. Therefore, the quest for comparatively simple, flexible, generic and highly accurate approximate analytical solutions continues. Although, the present of nonlinearities in the developed model makes it very difficult to develop exact analytical solutions to the problems under investigation, This paper was recommended for publication in revised form by Regional Editor Jaap Hoffman Hoffman

${ }^{1}$ Department of Mechanical Engineering, University of Lagos, Akoka, Lagos, Nigeria.

${ }^{*}$ E-mail address: mikegbeminiyiprof@yahoo.com

Manuscript Received 1 May 2017, Accepted 8 August 2017 
the classical way for finding exact analytical solution is obviously still very much important since it serves as an accurate benchmark for numerical and approximate analytical solutions. Also, exact analytical solutions are required to show the direct and precise relationship between the models parameters. When such exact analytical solutions are available, they provide good insights into the significance of various system parameters affecting the phenomena. Furthermore, most of the approximate analytical and purely numerical methods applied to solve nonlinear problems are shown to be computationally intensive. Exact analytical expression appears more appealing than the numerical solution as it helps to reduce the computation costs, simulations and task in the analysis of real life problems. It is more convenient for engineering calculations compare with numerical studies. Therefore, as a means of establishing an accurate benchmark for numerical or approximate analytical solutions, an exact analytical solution is required for the problem under investigation. Consequently, Abdel Kader et al. [27, 28, 29, 30] developed exact analytical solutions for fin problems with power-law and linear temperature-dependent thermal conductivity using symmetry reduction methods and partial Noether method, respectively. The concept of Noether's method and Nother's theorem as a systematic way of constructing conservation laws for di $\square$ erential equations with variational formulations was discovered by Noether [31]. The theorem provides the formula for the construction of the conservation laws for Euler-Lagrange equations once their Noether symmetries are known. The use of the theorem requires a Lagrangian in order to obtain the Noether symmetries and construct the first integrals. Also, the notions of partial Lagrangians, partial Noether operators and partial Euler-Lagrange equations are used in the construction of first integrals for ordinary di $\square$ erential equation that need not be derivable from variational principles [32-36].

In this present study, the Noether partial method is applied to develop exact analytical solutions to study the effects of magnetic field on the thermal performance of convective-radiative fin with linear temperaturedependent thermal conductivity. The exact analytical solutions in this study can serve as a starting point for a better understanding of the relationship between the physical quantities of the problems as it provides continuous physical insights into the problem than pure numerical or computation methods. Therefore, goal of this paper is to use partial Neother method to develop an exact analytical solution for the analysis thermal performance of convectiveradiative straight fin with temperature-dependent thermal conductivity in the presence of uniform magnetic field. Such an analytical tool is valuable as a design and optimization approach for large scale (not necessarily in size) finned heat exchangers where each fin/row are analytically analyzed and where the surrounding fluid is influenced by a magnetic field.

\section{Problem Formulation}

Consider a convective-radiative straight fin of temperature-dependent thermal conductivity $k(T)$, length L and thickness $\delta$ that is exposed on both faces to a convective environment at temperature $\mathrm{T}_{\mathrm{a}}$ and with heat transfer co-efficient $\mathrm{h}$ and subjected to magnetic field (such as in a liquid metal environment) as shown in Figure 1, assuming that the heat flow in the fin and its temperature remain constant with time, the temperature of the medium surrounding the fin is uniform, the fin base temperature is uniform, there is no contact resistance where the base of the fin joins the prime surface, also the fin thickness is small compared with its width and length, so that temperature gradients across the fin thickness and heat transfer from the edges of the fin may be neglected.

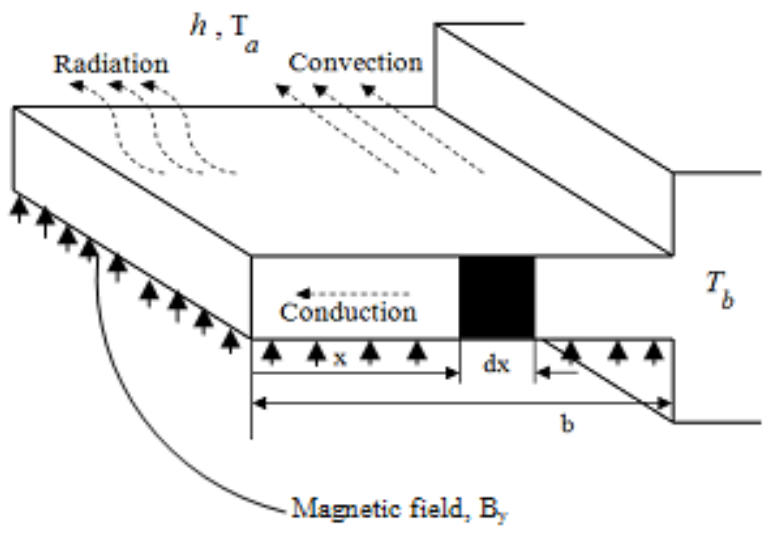

Figure 1. Schematic of the convective-radiative longitudinal porous fin with magnetic field 
The dimension $x$ pertains to the length coordinate which has its origin at the tip of the fin and has a positive orientation from the fin tip to the fin base. Based on the assumptions, the thermal energy balance could be expressed as stated in Eq. (1).

$$
q_{x}-\left(q_{x}+\frac{\delta q}{\delta x} d x\right)=h P\left(T-T_{a}\right) d x+\sigma_{s t} \varepsilon P\left(T^{4}-T_{a}^{4}\right) d x+\frac{J_{c} \times J_{c}}{\sigma} d x
$$

where

$$
J_{c}=\sigma(E+V \times B)
$$

As $d x \rightarrow 0$, Eq. (1) reduces

$$
-\frac{d q}{d x}=h P\left(T-T_{a}\right)+\sigma_{s t} \varepsilon P\left(T^{4}-T_{a}^{4}\right)+\frac{J_{c} \times J_{c}}{\sigma}
$$

From Fourier's law of heat conduction, the rate of heat conduction in the fin is given by

$$
q_{c}=-k(T) A_{c r} \frac{d T}{d x}
$$

where

$$
k(T)=k_{a}\left[1+\lambda\left(T-T_{a}\right)\right]
$$

Following Rosseland diffusion approximation, the radiation heat transfer rate is

$$
q_{r}=-\frac{4 \sigma_{s t} A_{c r}}{3 \beta_{R}} \frac{d T^{4}}{d x}
$$

Therefore, the total rate of heat transfer is given by

$$
q=-k(T) A_{c r} \frac{d T}{d x}-\frac{4 \sigma_{s t} A_{c r}}{3 \beta_{R}} \frac{d T^{4}}{d x}
$$

Substituting Eq. (7) into Eq. (3), we have

$$
\frac{d}{d x}\left(k_{a}\left[1+\lambda\left(T-T_{a}\right)\right] A_{c r} \frac{d T}{d x}+\frac{4 \sigma_{s t} A_{c r}}{3 \beta_{R}} \frac{d T^{4}}{d x}\right)=h P\left(T-T_{a}\right)+\sigma_{s t} \varepsilon P\left(T^{4}-T_{a}^{4}\right)+\frac{J_{c} \times J_{c}}{\sigma}
$$

Further simplification of Eq. (8) gives the governing differential equation for the fin as

$$
\frac{d}{d x}\left[\left[1+\lambda\left(T-T_{a}\right)\right] \frac{d T}{d x}\right]+\frac{4 \sigma_{s t}}{3 \beta_{R} k_{a}} \frac{d}{d x}\left(\frac{d T^{4}}{d x}\right)-\frac{h}{k_{a} t}\left(T-T_{a}\right)-\frac{\sigma_{s t} \varepsilon}{k_{a} t}\left(T^{4}-T_{a}^{4}\right)-\frac{J_{c} \times J_{c}}{\sigma k_{a} A_{c r}}=0
$$

The boundary conditions are

$$
\begin{aligned}
& x=0, \quad \frac{d T}{d x}=0, \\
& x=b, \quad T=T_{b}
\end{aligned}
$$

But 


$$
\frac{J_{c} \times J_{c}}{\sigma}=\sigma B_{o}^{2} u^{2}
$$

After substitution of Eq. (11) into Eq.(9), taking the magnetic field to vary linearly with temperature, we have

$$
\begin{aligned}
& \frac{d}{d x}\left[\left[1+\lambda\left(T-T_{a}\right)\right] \frac{d T}{d x}\right]+\frac{4 \sigma_{s t}}{3 \beta_{R} k_{a}} \frac{d}{d x}\left(\frac{d T^{4}}{d x}\right) \\
& -\frac{h}{k_{a} t}\left(T-T_{a}\right)-\frac{\sigma_{s t} \varepsilon}{k_{a} t}\left(T^{4}-T_{a}^{4}\right)-\frac{\sigma B_{o}^{2} u^{2}}{k_{\text {eff }} A_{c r}}\left(T-T_{a}\right)=0
\end{aligned}
$$

The case considered in this work is a situation where small temperature difference exists within the material during the heat flow. This actually necessitated the use of temperature-invariant physical and thermal properties of the fin. Also, it has been established that under such scenario, the term $\mathrm{T}^{4}$ can be expressed as a linear function of temperature. Therefore, we have

$$
T^{4} \cong 4 T_{a}^{3} T-3 T_{a}^{4}
$$

On substituting Eq. (13) into Eq. (12), we arrived at

$$
\begin{aligned}
& \frac{d}{d x}\left[\left[1+\lambda\left(T-T_{a}\right)\right] \frac{d T}{d x}\right]+\frac{16 \sigma_{s t}}{3 \beta_{R} k_{a}} \frac{d^{2} T}{d x^{2}} \\
& -\frac{h}{k_{a} t}\left(T-T_{a}\right)-\frac{4 \sigma_{s t} \varepsilon P T_{a}^{3}}{k_{a} t}\left(T-T_{a}\right)-\frac{\sigma B_{o}^{2} u^{2}}{k_{a} A_{c r}}\left(T-T_{a}\right)=0
\end{aligned}
$$

On introducing the following dimensionless parameters in Eq. (15) into Eq. (14),

$$
\begin{gathered}
\bar{x}=\frac{x}{b}, \theta=\frac{T-T_{a}}{T_{b}-T_{a}} \quad \beta=\lambda\left(T_{b}-T_{\infty}\right) \\
M^{2}=\frac{p b h}{A_{b} k_{a}} \quad R d=\frac{4 \sigma_{s t} T_{\infty}^{3}}{3 \beta_{R} k_{a}} \\
N=\frac{4 \sigma_{s t} b T_{\infty}^{3}}{k_{a}} \quad H=\frac{\sigma B_{0}^{2} u^{2}}{k_{a} A_{b}} .
\end{gathered}
$$

we arrived at the dimensionless form of the governing Eq. (16) as

$$
(1+4 R d) \frac{d^{2} \theta}{d \bar{x}^{2}}+\beta \theta \frac{d^{2} \theta}{d \bar{x}^{2}}+\beta\left(\frac{d \theta}{d \bar{x}}\right)^{2}-M^{2} \theta-N \theta-H \theta=0
$$

which gives

$$
\begin{aligned}
& \frac{d^{2} \theta}{d \bar{x}^{2}}+\frac{\beta}{(1+4 R d)} \theta \frac{d^{2} \theta}{d \bar{x}^{2}}+\frac{\beta}{(1+4 R d)}\left(\frac{d \theta}{d \bar{x}}\right)^{2} \\
& -\frac{M^{2}}{(1+4 R d)} \theta-\frac{N}{(1+4 R d)} \theta-\frac{H}{(1+4 R d)} \theta=0
\end{aligned}
$$

which can be written as 


$$
\frac{d^{2} \theta}{d \bar{x}^{2}}+\beta^{*} \theta \frac{d^{2} \theta}{d \bar{x}^{2}}+\beta^{*}\left(\frac{d \theta}{d \bar{x}}\right)^{2}-\left(M^{*}\right)^{2} \theta-N^{*} \theta-H^{*} \theta=0
$$

where

$$
\begin{aligned}
& \beta^{*}=\frac{\beta}{(1+4 R d)}, \quad\left(M^{*}\right)^{2}=\frac{M^{2}}{(1+4 R d)} \\
& N^{*}=\frac{N}{(1+4 R d)} \quad H^{*}=\frac{H}{(1+4 R d)} \theta
\end{aligned}
$$

and the dimensionless boundary conditions

$$
\begin{aligned}
\bar{x}=0, & \frac{d \theta}{d \bar{x}}=0 \\
& \bar{x}=1, \quad \theta=1
\end{aligned}
$$

\section{EXACT SOLUTION BY PARTIAL NOETHER METHOD}

Following the definition given by Kara et al. [31] and Naeem and Mahomed [32], a Lie operator $X$ of a form

$$
X=\xi \frac{\partial}{\partial \bar{x}}+\eta \frac{\partial}{\partial \theta}+\left(D_{\bar{x}} \eta-\theta^{\prime} D_{\bar{x}} \xi\right) \frac{\partial}{\partial \theta^{\prime}}+\ldots
$$

is a partial Noether operator corresponding to a partial Lagrangian $L \epsilon A$, if there exists a function $B \epsilon A, B \neq N L$ $+C, \mathrm{C}$ is a constant and $\mathrm{B}$ is gauge function, such that

$$
X(L)+L D_{\bar{x}}(\xi)=\left(\eta-\theta^{\prime} \xi\right) \frac{\delta L}{\delta \theta^{\prime}}+D_{\bar{x}}(B)
$$

Where $D_{x}$ is the the total differential with respect to $\mathrm{x}$ and $\frac{\delta}{\delta \theta^{\prime}}$ is the Euler-Langrange operator, $\frac{\delta}{\delta \theta^{\prime}}$ are given as

$$
\begin{gathered}
D_{\bar{x}}=\frac{\partial}{\partial \bar{x}}+\theta^{\prime} \frac{\partial}{\partial \theta}+\theta^{\prime \prime} \frac{\partial}{\partial \theta^{\prime}}+\ldots \\
\frac{\delta}{\delta \theta}=\frac{\partial}{\partial \theta}-D_{\bar{x}}\left(\frac{\partial}{\partial \theta^{\prime}}\right)+\ldots
\end{gathered}
$$

According to the Noether's theorem, If the Lie operator in Eq. (21) is a partial Noether operator of a partial Lagrangian $L \epsilon A$ corresponding to a partial Euler-Lagrange equation of Eq. (18) of the form $\frac{\delta L}{\delta \theta}=f^{\beta} E_{\beta}^{1}$, if and only if the characteristics $\left(\eta-\theta^{\prime} \xi\right) \in A$, of $\mathrm{X}$ is also the characteristics of the conservation law $D_{\bar{x}} I=0$, where the first integral I of the partial Euler-Lagrange equation of Eq. (20) is given by

$$
I=B-N(L)
$$

where, $\mathrm{N}$ is a Noether operator which is defined as: 


$$
N=\xi+\left(\eta-\theta^{\prime} \xi\right) \frac{\delta}{\delta \theta^{\prime}}+\ldots
$$

where,

$$
\frac{\delta}{\delta \theta^{\prime}}=\frac{\partial}{\partial \theta^{\prime}}-D_{\bar{x}}\left(\frac{\partial}{\partial \theta^{\prime \prime}}\right)+\ldots
$$

Again, following Kara et al. [31] and Naeem and Mahamed [32, 34, 36], consider the partial Langragian of Eq. (18)

$$
L=\frac{\left(\theta^{\prime}\right)^{2}}{2}
$$

where,

$$
\frac{\delta L}{\delta \theta}=-\theta^{\prime \prime}=\frac{\beta\left(\theta^{\prime}\right)^{2}-\left(\left(M^{*}\right)^{2}+N^{*}+H^{*}\right) \theta}{1+\beta \theta}
$$

In order to develop the partial Noether operator of Eq. (18), substitute Eq. (28) and (29) into the condition (22), we arrived at

$$
\begin{aligned}
& X\left(\frac{\left(\theta^{\prime}\right)^{2}}{2}\right)+\frac{\left(\theta^{\prime}\right)^{2}}{2} D_{\bar{x}}(\xi)=\left(\eta-\theta^{\prime} \xi\right) \\
& \left(\frac{\beta\left(\theta^{\prime}\right)^{2}-\left(\left(M^{*}\right)^{2}+N^{*}+H^{*}\right) \theta}{1+\beta \theta}\right)+D_{\bar{x}}(B)
\end{aligned}
$$

On substituting Eqs. (20) into Eq. (30), the determining equation as given in Eq. (31) was developed

$$
\begin{aligned}
& \left(D_{\bar{x}} \eta-\theta^{\prime} D_{\bar{x}} \xi\right) \theta^{\prime}+\frac{\left(\theta^{\prime}\right)^{2}}{2} D_{\bar{x}}(\xi)=\left(\eta-\theta^{\prime} \xi\right) \\
& \left(\frac{\beta\left(\theta^{\prime}\right)^{2}-\left(\left(M^{*}\right)^{2}+N^{*}+H^{*}\right) \theta}{1+\beta \theta}\right)+D_{\bar{x}}(B)
\end{aligned}
$$

If we let $\xi=\xi(\bar{x}, \theta), \quad \eta=\eta(\bar{x}, \theta)$ and $B=B(\bar{x}, \theta)$ [27], then Eq. (31) becomes

$$
\begin{aligned}
& -B_{\bar{x}}+\left(\frac{\left(\left(M^{*}\right)^{2}+N^{*}+H^{*}\right) \theta}{1+\beta \theta}\right) \eta+\left(-B_{\theta}-\frac{\left(\left(M^{*}\right)^{2}+N^{*}+H^{*}\right) \theta}{1+\beta \theta} \xi+\eta_{\bar{x}}\right) \theta^{\prime}+ \\
& +\left(\frac{\beta}{1+\beta \theta} \xi-\frac{1}{2} \xi_{\theta}\right)\left(\theta^{\prime}\right)^{3}+\left(-\frac{\beta}{1+\beta \theta} \eta+\eta_{\theta}-\frac{1}{2} \xi_{\bar{x}}\right)\left(\theta^{\prime}\right)^{2}=0
\end{aligned}
$$


Equating the coefficients of the derivatives of $\theta$ with zero, we obtain

$$
\begin{array}{ll}
\left(\theta^{\prime}\right)^{3}: & \frac{\beta}{1+\beta \theta} \xi-\frac{1}{2} \xi_{\theta} \\
\left(\theta^{\prime}\right)^{2}: & -\frac{\beta}{1+\beta \theta} \xi+\eta_{\theta}-\frac{1}{2} \xi_{\bar{x}} \\
\left(\theta^{\prime}\right): & -B_{\theta}-\frac{\left(\left(M^{*}\right)^{2}+N^{*}+H^{*}\right) \theta}{1+\beta \theta} \xi+\eta_{\bar{x}} \\
\left(\theta^{\prime}\right)^{0}: & -B_{\bar{x}}+\left(\frac{\left(\left(M^{*}\right)^{2}+N^{*}+H^{*}\right) \theta}{1+\beta \theta}\right) \eta
\end{array}
$$

It can easily be shown that the solutions of system of Eqs. (33)-(36) are given by

$$
\begin{aligned}
& \xi=6(1+\beta \theta)^{2}, \quad \eta=0, \\
& B=-\theta^{2}(3+2 \beta \theta)\left(\left(M^{*}\right)^{2}+N^{*}+H^{*}\right)
\end{aligned}
$$

Substituting Eqs. (28) into Eq. (27) and the resulting equation with the solutions in Eq. (36) gives

$$
I=-\theta^{2}(3+2 \beta \theta)\left(\left(M^{*}\right)^{2}+N^{*}+H^{*}\right)+3(1+\beta \theta)^{2}\left(\theta^{\prime}\right)^{2}
$$

If we take the first integral $I=c_{1}$, we obtain

$$
-\theta^{2}(3+2 \beta \theta)\left(\left(M^{*}\right)^{2}+N^{*}+H^{*}\right)+3(1+\beta \theta)^{2}\left(\theta^{\prime}\right)^{2}=c_{1}
$$

It should be noted that $\mathrm{c}_{1}$ is a constant. The constant $\mathrm{c}_{1}$ can be found using the using the boundary condition in Eq. (20),

$$
-\theta_{0}^{2}\left(3+2 \beta \theta_{0}\right)\left(\left(M^{*}\right)^{2}+N^{*}+H^{*}\right)=c_{1}
$$

where $\theta_{o}$ is the temperature of fin at the fin tip ( $(x=0)$, substiting (40) into (41), gives Eq. (42)

$$
\begin{aligned}
& \theta_{0}^{2}\left(3+2 \beta \theta_{0}\right)\left(\left(M^{*}\right)^{2}+N^{*}+H^{*}\right)-\theta^{2}(3+2 \beta \theta) \\
& \left(\left(M^{*}\right)^{2}+N^{*}+H^{*}\right)+3(1+\beta \theta)^{2}\left(\theta^{\prime}\right)^{2}=0
\end{aligned}
$$


For the purpose of achieving a straight-forward integration, let

$$
\theta=g(\bar{x})-\frac{1}{\beta}
$$

Substituting Eq. (41) into Eq. (40), we arrived at

$$
\begin{aligned}
& {\left[g^{\prime}(\bar{x})\right]^{2}=\frac{\left(\left(M^{*}\right)^{2}+N^{*}+H^{*}\right)}{3 \beta^{4}[g(\bar{x})]^{2}}} \\
& \left(\left(-\left(1+\beta \theta_{0}\right)^{2}\left(2 \beta \theta_{0}-1\right)+2 \beta^{3} g(\bar{x})^{3}-3 \beta^{2} g(\bar{x})^{2}\right)\right)
\end{aligned}
$$

The integration of Eq. (43) gives

$$
\int_{0}^{1} d \bar{x}=\frac{-\sqrt{3} \beta^{2}}{\sqrt{\left(\left(M^{*}\right)^{2}+N^{*}+H^{*}\right)}} \int_{\theta_{0}+\frac{1}{\beta}}^{\theta+\frac{1}{\beta}}\left(\begin{array}{l}
\left(-\left(1+\beta \theta_{0}\right)^{2}\right. \\
\left.\left(2 \beta \theta_{0}-1\right)+2 \beta^{3}[g(\bar{x})]^{3}-3 \beta^{2}[g(\bar{x})]^{2}\right)
\end{array}\right)^{-\frac{1}{2}} d g
$$

On carrying out the integration in Eq. (44), the exact implicit solution of Eq. (20) is obtained as given in Eq. (45)

$$
\bar{x}=-\frac{\sqrt{3}}{\sqrt{-2 \gamma\left(\left(M^{*}\right)^{2}+N^{*}+H^{*}\right)}}\left(\begin{array}{l}
\gamma E\left(\sin ^{-1}\left(\frac{1}{\sqrt{2} \sqrt[4]{3}} \sqrt{\rho-\frac{4 \beta}{\alpha} \theta_{o}}\right), \frac{2 \sqrt{3} \alpha}{\gamma}\right) \\
-\gamma E\left(\sin ^{-1}\left(\frac{1}{\sqrt{2} \sqrt[4]{3}} \sqrt{\rho-\frac{4 \beta}{\alpha} \theta}\right), \frac{2 \sqrt{3} \alpha}{\gamma}\right) \\
+4\left(1+\beta \theta_{0}\right)\left(\sin ^{-1}\left(\frac{1}{\sqrt{2} \sqrt[4]{3}} \sqrt{\rho-\frac{4 \beta}{\alpha} \theta_{o}}\right), \frac{2 \sqrt{3} \alpha}{\gamma}\right) \\
-F\left(\sin ^{-1}\left(\frac{1}{\sqrt{2} \sqrt[4]{3}} \sqrt{\rho-\frac{4 \beta}{\alpha}} \theta\right), \frac{2 \sqrt{3} \alpha}{\gamma}\right)
\end{array}\right)
$$

The unknown parameter $\theta_{o}$ in Eq. (45) is determined using the boundary condition $\theta(1)=1$. i.e. 


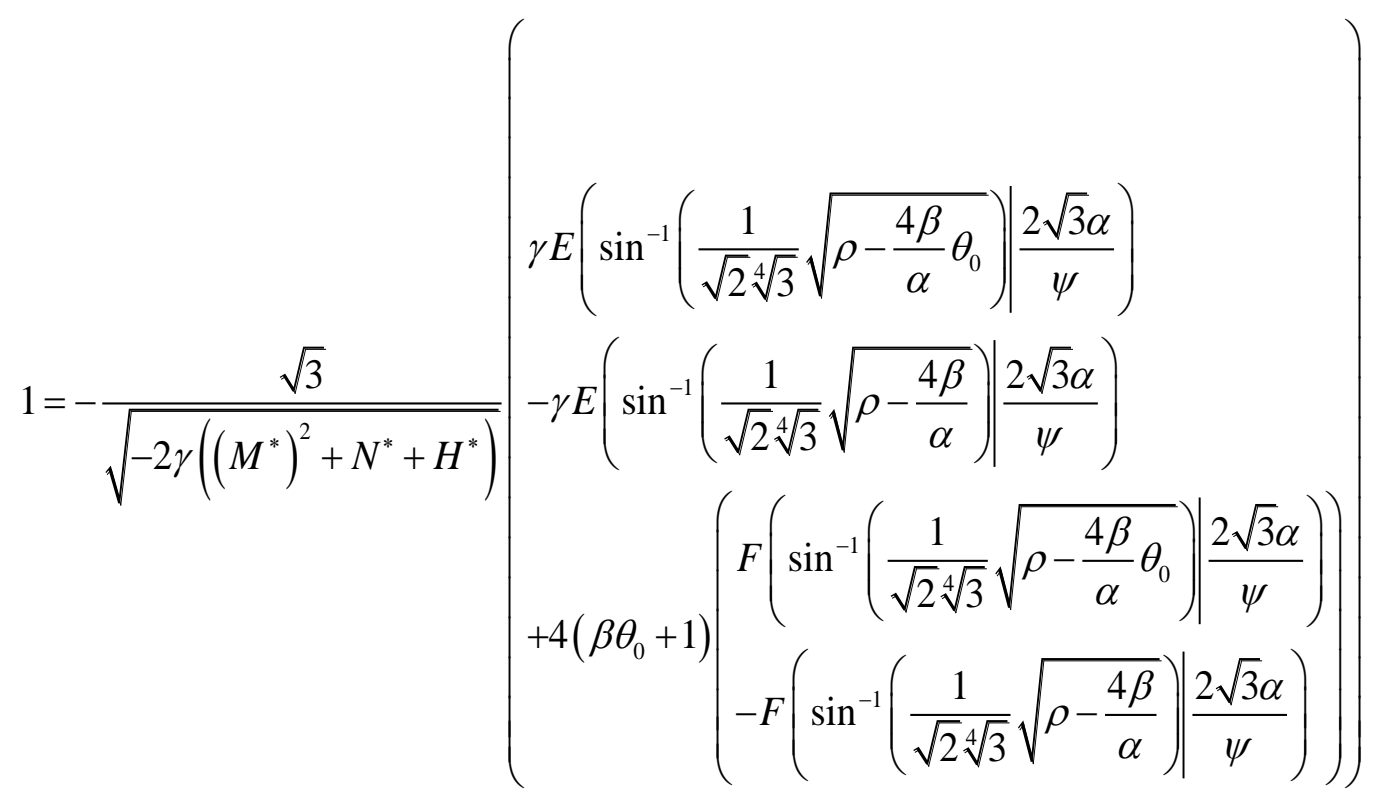

where, $E$ is the incomplete elliptic integral of the second kind with modulus $m^{2}$ and amplitude $\phi$.

$$
E(\phi, K)=\int_{0}^{\phi} \sqrt{1-K^{2} \sin ^{2} \vartheta} d \vartheta
$$

$F$ is the incomplete elliptic integral of the first kind with modulus $m^{2}$ and amplitude $\phi$.

$$
F(\phi, K)=\int_{0}^{\phi} \frac{1}{\sqrt{1-K^{2} \sin ^{2} \vartheta}} d \vartheta
$$

and

$$
\begin{gathered}
\alpha=\sqrt{3-4 \beta u_{0}-4 \beta^{2} u_{0}^{2}} \\
\gamma=-3 \sqrt{3 a}-6 \beta u_{0} \\
\rho=\sqrt{3}-\frac{1}{\alpha}\left(3+2 u_{0} \beta\right)
\end{gathered}
$$

\section{ANALYTICAL EVALUATION OF THE INCOMPLETE ELLIPTIC INTEGRALS}

For the incomplete elliptic integral of the first kind

$$
F(\phi, K)=\int_{0}^{\phi} \frac{d \vartheta}{\sqrt{1-K^{2} \sin ^{2} \vartheta}}
$$

In order to evaluate the integral, we expand the integral in the form

$$
\begin{aligned}
& \frac{1}{\sqrt{1-K^{2} \sin ^{2} \vartheta}}=1+\frac{K^{2}}{2} \sin ^{2} \vartheta+ \\
& \frac{3 K^{4}}{8} \sin ^{4} \vartheta+\frac{5 K^{6}}{16} \sin ^{6} \vartheta+\frac{35 K^{8}}{128} \sin ^{8} \vartheta+\ldots
\end{aligned}
$$


The above Eq. (51) can written as

$$
\begin{aligned}
& \frac{1}{\sqrt{1-K^{2} \sin ^{2} \vartheta}}-1=\frac{K^{2}}{2} \sin ^{2} \vartheta+\frac{3 K^{4}}{8} \sin ^{4} \vartheta \\
& +\frac{5 K^{6}}{16} \sin ^{6} \vartheta+\frac{35 K^{8}}{128} \sin ^{8} \vartheta+\ldots+\left(\prod_{n=1}^{N}\left(\frac{2 n-1}{2 n}\right)\right) K^{2 n} \sin ^{2 n} \vartheta
\end{aligned}
$$

Generally, we can write

$$
\frac{1}{\sqrt{1-K^{2} \sin ^{2} \vartheta}}=1+\sum_{n=1}^{N}\left(\prod_{n=1}^{N} \frac{2 n-1}{2 n}\right) K^{2 n} \sin ^{2 n} \vartheta
$$

The above series is uniformly convergent for all $\vartheta$, and may, therefore, be integrated term by term. Then, we have

$$
F(\phi, K)=\int_{0}^{\phi}\left\{1+\sum_{n=1}^{N}\left(\prod_{n=1}^{N} \frac{2 n-1}{2 n}\right) K^{2 n} \sin ^{2 n} \vartheta\right\} d \vartheta
$$

But

$$
\int \sin ^{2 n} \vartheta d \vartheta=\frac{-\cos \vartheta}{2 n}\left\{\begin{array}{l}
\sin ^{2 n-1} \vartheta+ \\
\sum_{k=1}^{n-1} \frac{(2 n-1)(2 n-3) \ldots(2 n-2 k+1)}{2^{k}(n-1)(n-2) \ldots(n-k)} \sin ^{2 n-2 k-1} \vartheta
\end{array}\right\}+\frac{(2 n-1) ! !}{2^{n} n !} \vartheta
$$

Therefore

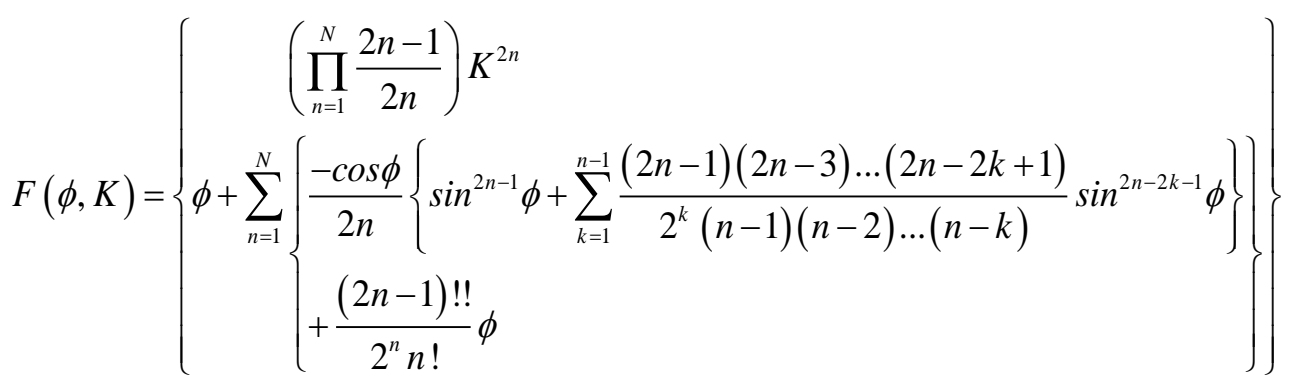

In the same way, the analytical evaluation of the incomplete elliptic integral of second kind can be found. The simple expanded form of the first two terms of Eq. (56) is given as

$$
F(\phi, K)=\left\{\begin{array}{l}
\phi+\frac{1}{2} K^{2}\left[\left(\frac{-\cos \phi \sin \phi}{2}-\frac{\phi \cos \phi}{4}+\frac{1 ! !}{2} \phi\right)\right] \\
+\left(\frac{1}{2} \cdot \frac{3}{4} \cdot K^{4}\left\{\frac{-\cos \phi}{4}\left\{\sin ^{3} \phi+\frac{3}{2} \sin \phi\right\}+\frac{1 ! !}{2} \phi+\frac{3 ! !}{2^{2} 2 !} \phi\right\}\right)+\ldots
\end{array}\right\}
$$

\section{RESULTS AND DISCUSSION}

Figures 2 and 3 show the effects thermo-geometric term, radiation number and magnetic number (Hartmann number) on the dimensionless temperature distribution of the fin. The figures depict the effects thermogeometric, 


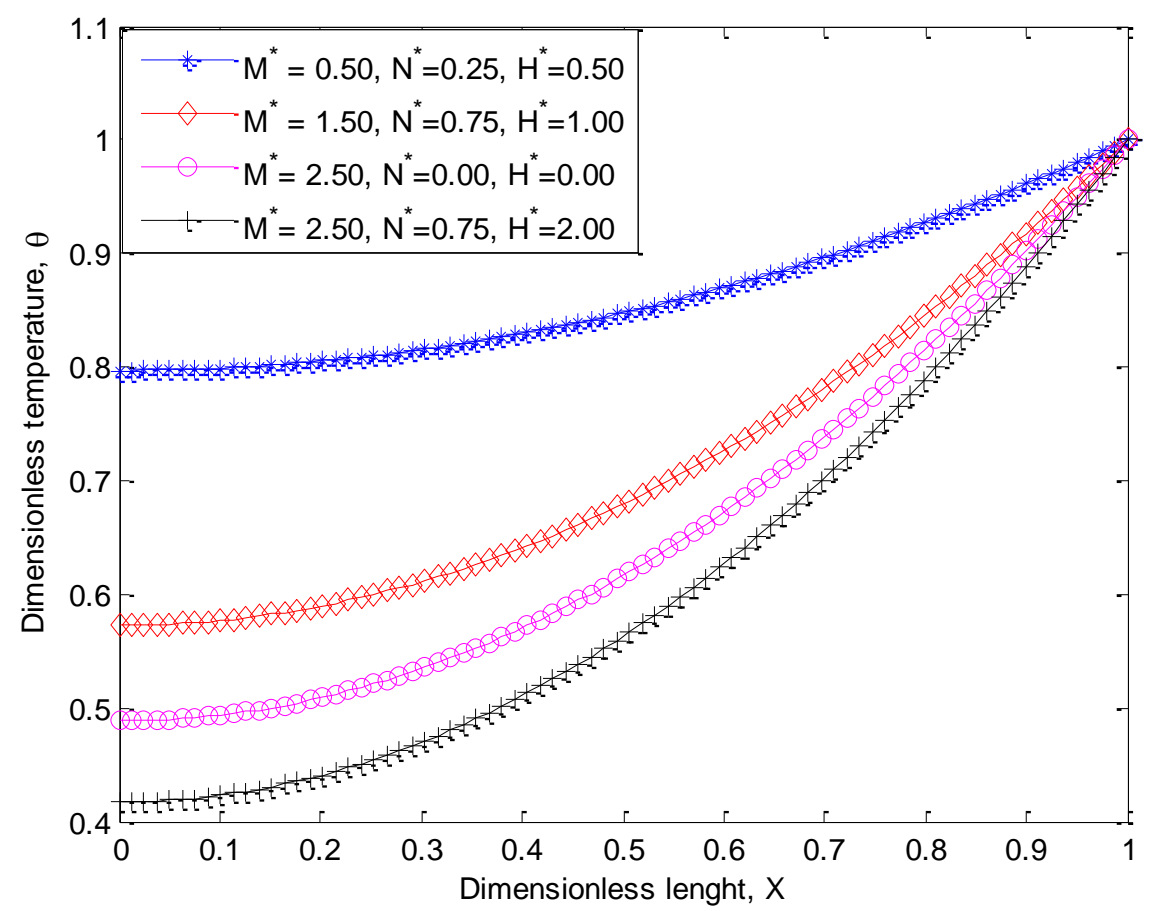

Figure 2. Effects of radiation and Hartmann numbers on the temperature distribution in the fin when $\beta=0.5$

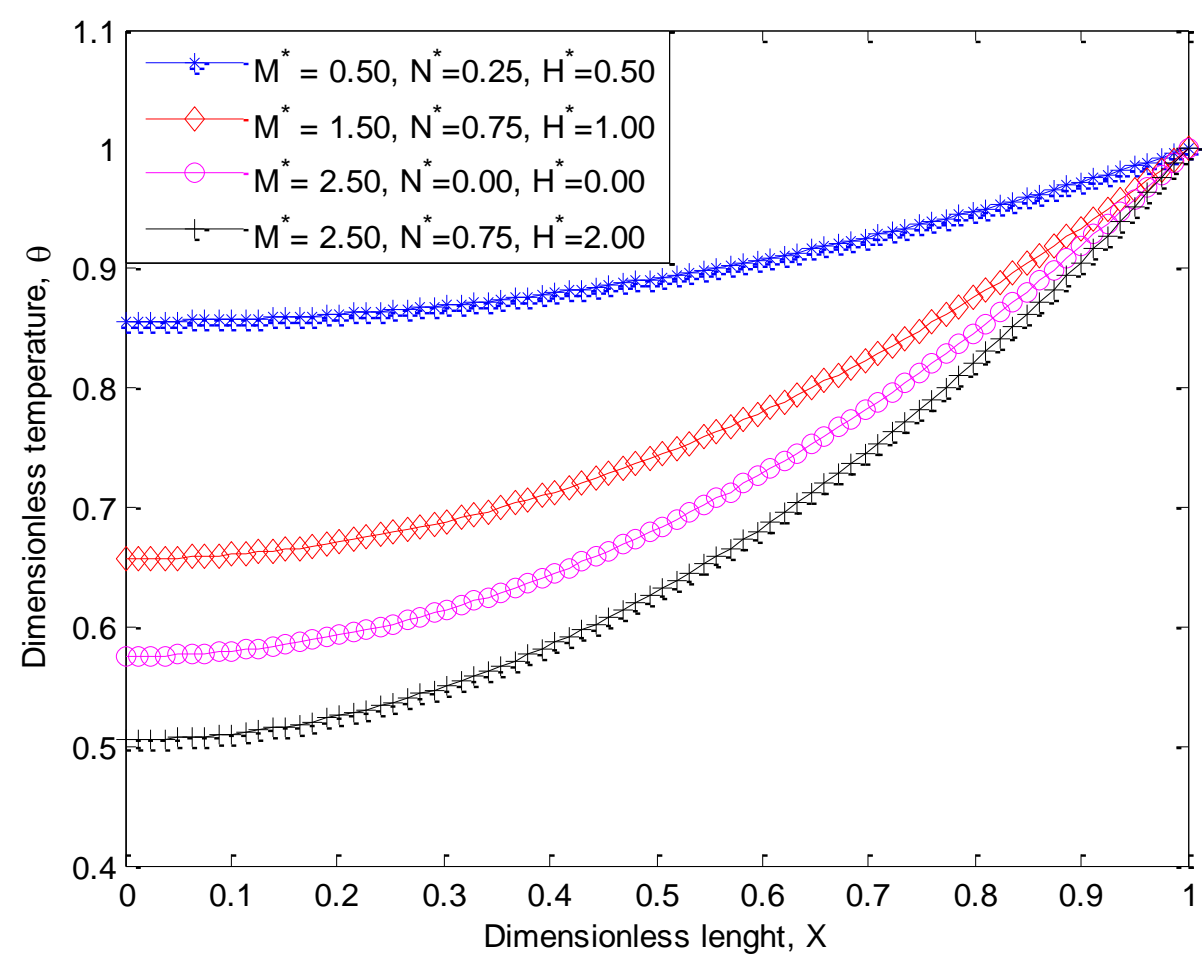

Figure 3. Effects of radiation and Hartmann numbers on the temperature distribution in the fin when $\beta=1.5$

magnetic and nonlinear parameter temperature-dependent thermal conductivity term on the thermal performance of the fin. From the figures, it could be deducted that the nonlinear thermal conductivity parameter has direct and significant effects on the rate of heat transfer of the fin.

Figs. 4 and 5 show the effects of non-linear thermal conductivity on the fin thermal performance under different thermo-geometric parameter, radiation number and Hartmann number. The results show that performance of the 


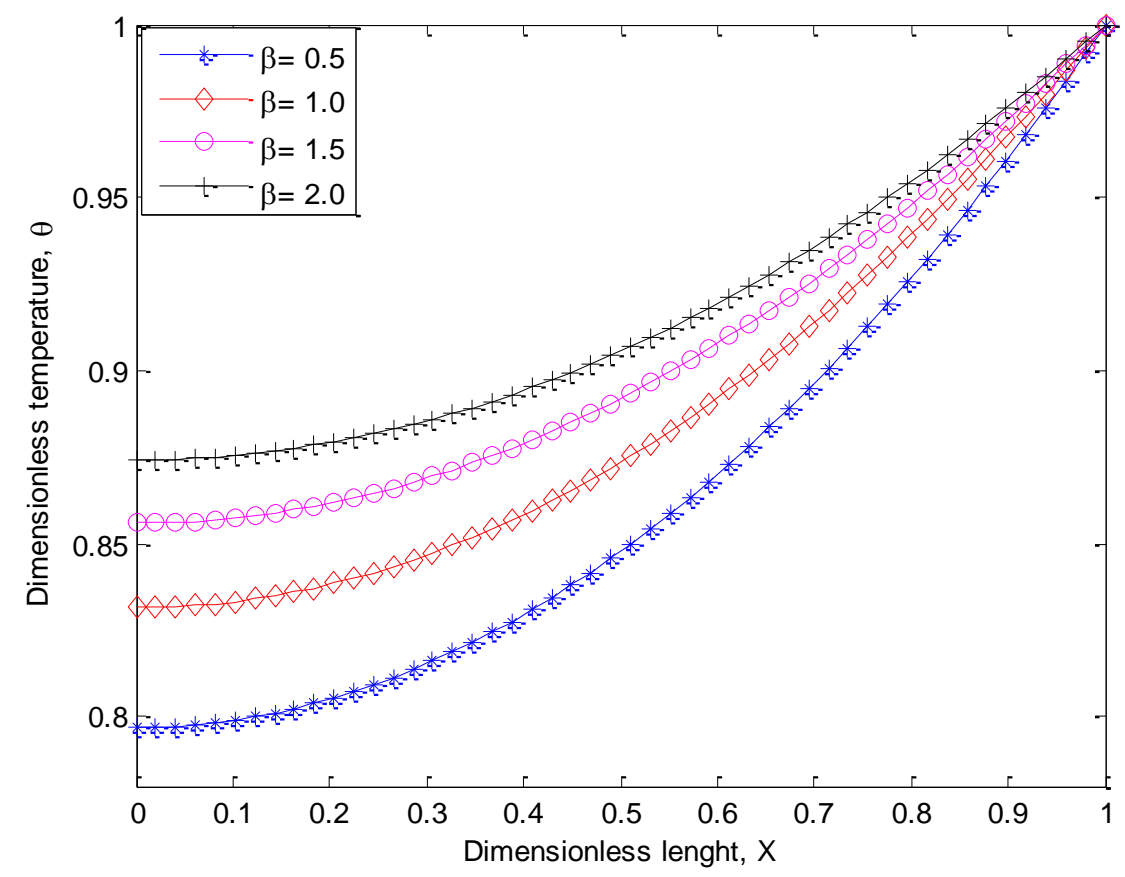

Figure 4. Effects of non-linear parameter on the temperature distribution in the fin when $\mathrm{M}=0.50, \mathrm{~N}=0.25$, $\mathrm{H}=0.50$

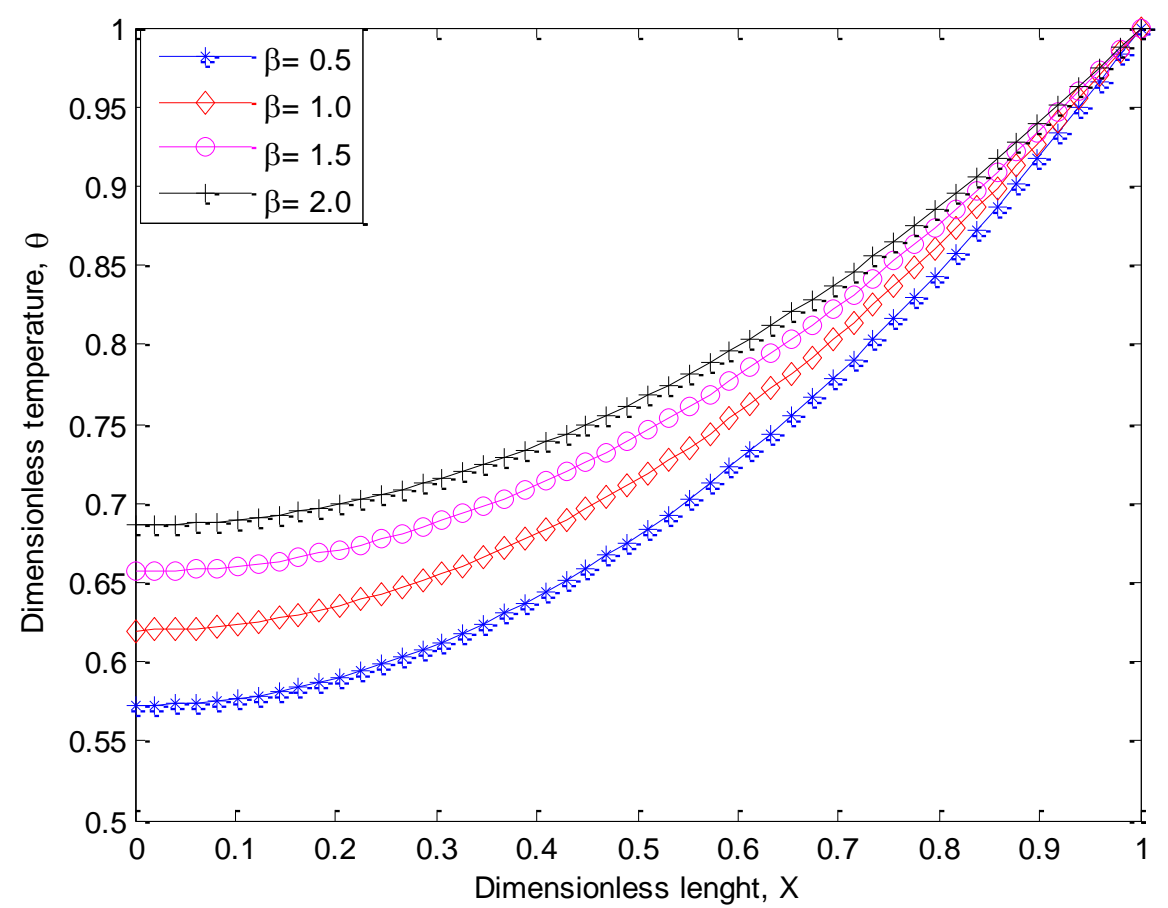

Figure 5. Effects of non-linear parameter on the temperature distribution in the fin when $\mathrm{M}=1.50, \mathrm{~N}=0.75$,

$$
\mathrm{H}=1.00
$$

fin temperature decreases with increase in the nonlinear term or the thermal conductivity number. Also, the figures depict that as the thermo-geometric parameter increases, the rate of heat transfer through the fin increases as the temperature in the fin drops faster.

Fig. 6 shows the influence of magnetic parameter, Hartmann number on the thermal performance of the fin. From the figures, it shows that as the magnetic parameter increases, the temperature decreases rapidly and the rate of heat transfer (the convective-radiative heat transfer) through the fin increases as implied in the figures. The 


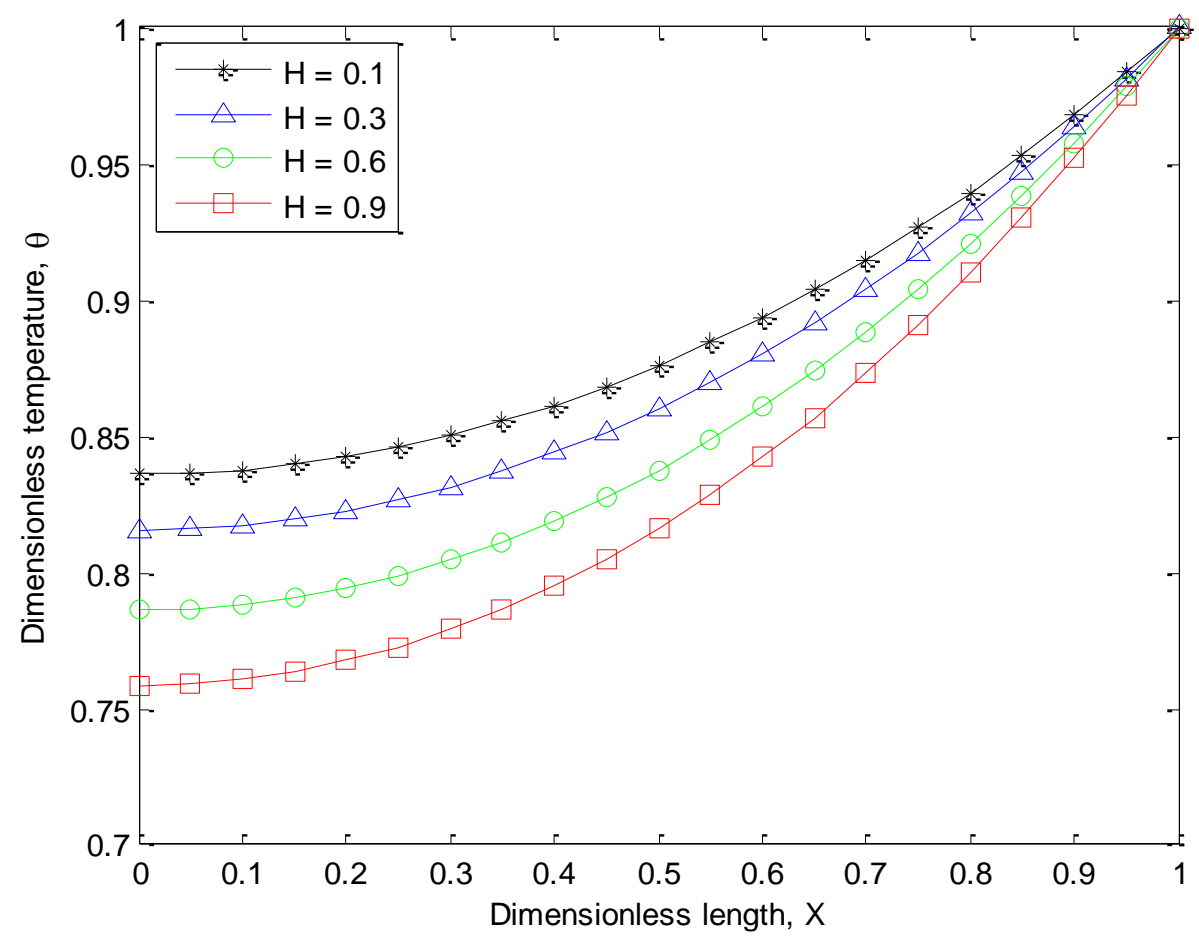

Figure 6. Effects of magnetic parameter on the temperature distribution in the fin

rapid decrease in fin temperature due to increase in the magnetic parameter is because as Hartmann number increases, the magnetic force increases and this in turn increases the magnetic field strength which consequently decreases the temperature of the fin as shown in the figures. Therefore, the thermal performance of the fin is increased by the presence of the magnetic field. This is in agreement with the deduction from works of Razazadeh et al. [37] and Hoshyar et al. [38].

Table 1. Comparison of results when $\beta=0.00, \mathrm{M}=1.00, \mathrm{~N}=0.00, \mathrm{H}=0.00$

\begin{tabular}{|lllllc|}
\hline$X$ & NM & $\begin{array}{c}\text { ADM } \\
{[5]}\end{array}$ & $\begin{array}{c}\text { HPM } \\
{[9]}\end{array}$ & $\begin{array}{c}\text { GMWR } \\
{[19]}\end{array}$ & $\begin{array}{c}\text { PNM } \\
\text { (The present study) }\end{array}$ \\
\hline 0.0 & 0.648054 & 0.648054 & 0.648054 & 0.648054 & 0.648054 \\
0.1 & 0.651297 & 0.651297 & 0.651297 & 0.651297 & 0.651297 \\
0.2 & 0.661059 & 0.661059 & 0.661059 & 0.661057 & 0.661057 \\
0.3 & 0.677436 & 0.677436 & 0.677436 & 0.677436 & 0.677436 \\
0.4 & 0.700594 & 0.700594 & 0.700594 & 0.700594 & 0.700594 \\
0.5 & 0.730763 & 0.730763 & 0.730763 & 0.730763 & 0.730763 \\
0.6 & 0.768246 & 0.768246 & 0.768246 & 0.768246 & 0.768246 \\
0.7 & 0.813418 & 0.813418 & 0.813418 & 0.813418 & 0.813418 \\
0.8 & 0.866731 & 0.866731 & 0.866731 & 0.866731 & 0.866731 \\
0.9 & 0.928718 & 0.928718 & 0.928718 & 0.928718 & 0.928718 \\
1.0 & 1.000000 & 1.000000 & 1.000000 & 1.000000 & 1.000000 \\
\hline
\end{tabular}

The Table 1 shows the comparison of past results in literatures using other methods with the present study using partial Neother method (PNM). From the table, it shows that the results of partial Neother method agree excellently with both the numerical method (NM) results and the results of Adomian decomposition method (ADM), Homotopy perturbation method (HPM) and Galerkin's method of weighted residual (GMWR) as found in literatures.

The physical meaning of $\beta=0.00, \mathrm{M}=1.00, \mathrm{~N}=0.00, \mathrm{H}=0.00$ is a case of convective fin with invariant thermal conductivity and where there is no influence of radiative and magnetic field. 


\section{CONCLUSION}

In this work, effects of magnetic field, radiation, thermo-geometric and the nonlinear thermal conductivity parameters on the thermal performance of the fin have been investigated through the developed exact analytical solution using partial Noether method. From the analysis, it shows that the thermal performance of the fin increases as the magnetic, radiative and thermo-geometric parameters increase. The exact analytical solution can serve as an accurate benchmark for numerical and approximate analytical solutions as it shows the direct and precise relationship between the models parameters. Also, such an analytical tool is valuable as a design and optimization approach for large scale (not necessarily in size) finned heat exchangers where each fin/row are analytically analyzed and where the surrounding fluid is influenced by a magnetic field.

\section{NOMENCLATURE}

$A_{\text {cr }}$ cross sectional area of the fins

$A_{p}$ profile area of the fins

$b$ length of the fin

B magnetic induction

$\mathrm{B}_{\mathrm{o}}$ magnetic field intensity

E electric field

$\mathrm{h}$ heat transfer coefficient

H Hartmann number

$\mathrm{J}$ total current intensity

$\mathrm{J}_{\mathrm{c}} \quad$ conduction current intensity

$\mathrm{k}$ thermal conductivity of the fin material

$\mathrm{k}_{\mathrm{a}}$ thermal conductivity of the fin material at ambient temperature

$\mathrm{M}$ dimensionless thermo-geometric fin parameter

$\mathrm{m}^{2}$ thermo-geometric fin parameter

$\mathrm{N}$ dimensionless radiation number

q total rate of heat transfer

$\mathrm{q}_{\mathrm{c}}$ rate of heat conduction transfer

$\mathrm{q}_{\mathrm{r}}$ rate of heat radiation transfer

Ra Modified Rayleigh number

$\mathrm{Rd}$ radiation-conduction number

$\mathrm{P}$ perimeter of the fin

$t$ thickness of the fin

$\mathrm{T}$ temperature

$\mathrm{T}_{\mathrm{a}} \quad$ ambient temperature

$\mathrm{T}_{\mathrm{b}} \quad$ temperature at the base of the fin

$\mathrm{V}$ voltage

$\mathrm{x}$ fin axial distance, $\mathrm{m}$

$\mathrm{X}$ dimensionless length of the fin

$\lambda$ non-linear thermal conductivity parameter

$\beta \quad$ dimensionless non-linear thermal conductivity parameter

$\beta_{\mathrm{R}} \quad$ Rosseland extinction coefficient

$\theta$ dimensionless temperature

$\varepsilon \quad$ emmisivity of the fin

$\sigma \quad$ electric conductivity

$\sigma_{\text {st }} \quad$ Stefan-Boltmann constant

\section{REFERENCES}

[1] Aziz, A. Enamul-Huq.S. (1973). Perturbation solution for convecting fin with temperature dependent thermal conductivity, Journal of Heat Transfer 97, 300-301.

[2] Aziz, A., (1977). Perturbation solution for convecting fin with internal heat generation and temperature dependent thermal conductivity, International Journal of Heat and Mass Transfer, 1253-5. 
[3] Campo, A. Spaulding, R. J., (1999). Coupling of the methods of successive approximations and undetermined coefficients for the prediction of the thermal behaviour of uniform circumferential fins," Heat and Mass Transfer, 34 (6), 461-468.

[4] Chiu, C. H., Chen, C. K.. (2002). A decomposition method for solving the convective longitudinal fins with variable thermal conductivity, International Journal of Heat and Mass Transfer 45, 2067-2075.

[5] Arslanturk, A. (2005). A decomposition method for fin efficiency of convective straight fin with temperature dependent thermal conductivity, International Communications in Heat and Mass Transfer Transfer, 32(6), 831841.

[6] Ganji, D. D. 2006. The application of He's homotopy perturbation method to nonlinear equations arising in heat transfer, Physics Letters A A, 355, 337-341.

[7] He, J.H. (1999). Homotopy perturbation method, Computer Methods in Applied Mechanics and Engineering, $178,257-262$.

[8] Chowdhury M. S. H., Hashim I. (2008). Analytical solutions to heat transfer equations by homotopyperturbation method revisited, Physical Letters A, 372, 1240-1243.

[9] Rajabi, A. (2007). Homotopy perturbation method for fin efficiency of convective straight fins with temperature dependent thermal conductivity .Physics Letters A364, 33-37.

[10] Chowdhury,M. S. H., Hashim, I. Abdulaziz, O. (2009). Comparison of homotopy analysis method and homotopy-perturbation method for purely nonlinear fin-type problems, Communications in Nonlinear Science and Numerical Simulation 14, 371-378.

[11] Mustafa, I. (2008). Application of Homotopy analysis method for fin efficiency of convective straight fin with temperature dependent thermal conductivity. Mathematics and Computers Simulation 79, $189-200$.

[12] Hosseini, K. Daneshian, B. Amanifard, N. Ansari, R. (2012). Homotopy Analysis Method for a Fin with Temperature Dependent Internal Heat Generation and Thermal Conductivity, International Journal of Nonlinear Science. 14(2), 201-210.

[13] Domairryand, G. Fazeli, M. (2009). Homotopy analysis method to determine the fin efficiency of convective straight fins with temperature dependent thermal conductivity. Communication in Nonlinear Science and Numerical Simulation 14, pp. 489-499.

[14] Coskun, S. B. and Atay, M. T. (2007). analysis of convective straight and radial fins with temperature dependent thermal conductivity using variational iteration method with comparision with respect to finite element analysis. Mathematical Problem in Engineering, 15.

[15] Languri, E. M. Ganji, D. D. Jamshidi, N. (2008). Variational Iteration and Homotopy perturbation methods for fin efficiency of convective straight fins with temperature dependent thermal conductivity. 5th WSEAS Int .Conf .On FLUID MECHANICS (fluids 08) Acapulco, Mexico January 25 -27.

[16] Coskun, S. B. and Atay, M. T. (2008). Fin efficiency analysis of convective straight fin with temperature dependent thermal conductivity using variational iteration method, Applied Thermal Engineering, 28, $2345-2352$.

[17] Atay, M. T. Coskun. S. B. (2008). Comparative analysis of power-law fin-type problems using variational iteration method and finite element method, Mathematical Problems in Engineering.

[18] Khani, F. Ahmadzadeh,R.M. HamediNejad, H. (2009). Analytical solutions and efficiency of the nonlinear fin problem with temperature-dependent thermal conductivity and heat transfer coefficient, Communications in Nonlinear Science and Numerical Simulation 14 (8), 3327-3338.

[19] Sobamowo. M. G. (2016). Thermal analysis of longitudinal fin with temperature-dependent properties and internal heat generation using Galerkin's method of weighted residual. Applied Thermal Engineering 99, 13161330

[20] Joneidi.,A. A.,Ganji, D. D. Babaelahi. M. (2009). Differential transformation method to determine fin efficiency of convective straight fins with temperature dependent thermal conductivity. International Communication in Heat and Mass transfer 36, 757-762

[21] Moradi, M. Ahmadikia, H. (2010). Analytical Solution for different profiles of fin with temperature dependent thermal conductivity. Hindawi Publishing Corporation Mathematical Problem in Engineering.

[22] Mosayebidorcheh, Ganji, D. D. Farzinpoor. M. (2014). Approximate Solution of the nonlinear heat transfer equation of a fin with the power-law temperature-dependent thermal conductivity and heat transfer coefficient, Propulsion and Power Research, 41-47.

[23] Ghasemi,S. E. Hatami, M. Ganji, D. D. (2014). Thermal analysis of convective fin with temperaturedependent thermal conductivity and heat generation, Cases Studies in Thermal Engineering, 4, 1-8.

[24] Ganji, D. D. Dogonchi, A. S. (2014). Analytical investigation of convective heat transfer of a longitudinal fin with temperature-dependent thermal conductivity, heat transfer coefficient and heat generation, International Journal of Physical Sciences, 9 (21), 466-474.

[25] Fernandez, A. (2009). On some approximate methods for nonlinear models. Applied Mathematics and Computation, 215 (1), 168-74.

[26] Aziz, A. and Bouaziz, M. N. (2011). A least squares method for a longitudinal fin with temperature dependent internal heat generation and thermal conductivity, Energy Conversion and Management, 52, 2876-2882. 
[27] Abdel Latif M.S., Abdel Kader A.H., Nour H.M. (2015). Exact implicit solution of nonlinear heat transfer in rectangular straight fin using symmetry reduction methods, Applications And Applied Mathematics-An International Journal., 10 (2),864-877.

[28] Abdel Kader A.H., Abdel Latif M.S., Nour H.M. (2016). General exact solution of the fin problem with variable thermal conductivity, Propulsion and Power Research, 5(1), 63-69.

[29] Abdel Kader A.H., Abdel Latif M.S., Nour H.M. (2016). General exact solution of the fin problem with the power law temperature - dependent thermal conductivity, Mathematical Methods in the Applied Sciences, 39 (6), 1513-1521.

[30]Abdel Kader .A, H. Abdel Latif, M. S. and Nour, H. M. (2016). Exact solution of fin problem with linear temperature-dependent thermal conductivity. Journal of Applied Mathematics and Computational Mechanics, 15(4), 51-61.

[31] Kara, A. H. , Mahomed F. M., Naeem I, W. C. Sofo. (2007). Partial Noether and the first integrals via partial Lagrangian. Mathematical Methods in the Applied Sciences, 30 (16), 2079-2089.

[32] Naheem I., Mahomed F. M. (2008). Noether, partial Neother operators and the first integrals for a linear system. Journal of Mathematical Analysis and Applications., 342, 70-82.

[33] Noether, E. (1918) Invariant Variations probleme, Nachr. K“onig Gesell Wissen, G“ottingen’, Math. Phys. K1. Heft 2, 235-257. (English translation in Transport Theory and Statical Physics 1971 1, 186-207.)

[34]Naeem I and Mahomed F M (2008) Partial Noether operators and first integrals for a system with two degrees of freedom, Journal of Nonlinear Mathematical Physics, 15, 165-178.

[35]Naeem I and Mahomed F M (2008) Noether, partial Noether operators and first integrals or a linear system, Journal of Mathematical Analysis and Applications, 342, 70-82.

[36] Naeem, I., Mahomed, F. M. (2009). Approximate partial Noether operators and first integrals for coupled nonlinear oscillators. Nonlinear Dynamics, 57(1-2), 303-311.

[37] Amirkolaei, R. S., Ganji, D. D. Slarian H. (2014). Determination of temperature distribution for porous fin which is exposed to uniform magnetic field to a vertical isothermal surface by homotopy analysis method and collocation method. Indian Journal of Scientific Research, 1(2), 215-222.

[38] Hoshyar, H., Ganji, D. D. Majidian, A. R. (2016). Least square method for porous fin in the presence of uniform magnetic field. Journal of Applied Fluid Mechanics, 9 (2), 661-668. 